\title{
Right-lateralized intrinsic brain dynamics predict monitoring abilities
}

\author{
Ettore Ambrosini ${ }^{1,2,3} \cdot$ Mariagrazia Capizzi $^{1} \cdot$ Sandra Arbula ${ }^{1,4} \cdot$ Antonino Vallesi $^{1,3,5}$ (i)
}

Published online: 27 January 2020

(C) The Psychonomic Society, Inc. 2020

\begin{abstract}
Intrinsic brain dynamics may play an important role in explaining interindividual variability in executive functions. In the present electroencephalography (EEG) study, we focused on the brain lateralization patterns predicting performance on three different monitoring tasks of temporal, verbal, and spatial nature. These tasks were administered to healthy young participants after their EEG was recorded during a resting state session. Behavioral indices of monitoring efficiency were computed for each task and a source-based spectral analysis was performed on participants' resting-state EEG activity. A lateralization index was then computed for each of 75 homologous cortical regions as the right-left difference score for the log-transformed power ratio between beta and alpha frequencies. Finally, skipped Pearson correlations between the lateralization index in each cortical region and behavioral performance of the three monitoring tasks were computed. An intersection among the three tasks showed that rightlateralization in different prefrontal regions, including the middle frontal gyrus, was positively correlated with monitoring abilities across the three tasks. In conclusion, right-lateralized brain mechanisms set the stage for the ability to monitor for targets in the environment, independently of the specific task characteristics. These mechanisms are grounded in hemispheric asymmetry dynamics already observable at rest.
\end{abstract}

Keywords Resting-state EEG $\cdot$ Hemispheric asymmetries $\cdot$ Prefrontal cortex $\cdot$ Temporal preparation $\cdot$ Monitoring $\cdot$ Foreperiod effect

\section{Introduction}

The environment in which we live is crowded with all sorts of stimuli, among which we should be able to detect those that are critical to our daily activities and goals. One of the most important tasks faced by our brain is to check for the occurrence of such stimuli by evaluating their conditional probabilities and likely timing of occurrence. Moreover, in order to

Ettore Ambrosini

ettore.ambrosini@unipd.it

Antonino Vallesi

antonino.vallesi@unipd.it

1 Department of Neuroscience \& Padova Neuroscience Center, University of Padova, Padova, Italy

2 Department of General Psychology, University of Padova, Padova, Italy

3 Department of Neuroscience, University of Padova, Padova, Italy

4 Area of Neuroscience, SISSA, Trieste, Italy

5 Neuroscience Research Unit, IRCCS San Camillo Hospital, Venice, Italy control and optimize our behavior effectively, our brain also evaluates the relative status of these stimuli in relation to each other and, most importantly, with respect to our intended plans and goals. These cognitive abilities collectively go under the name of monitoring.

Monitoring has been conceptualized as a set of "qualitycheck" processes that allow individuals to optimize performance. For example, monitoring is required when we evaluate the progress of our actual weight loss toward an ideal planned weight (Benn et al., 2014), when we search for a red flag surrounded by other colored flags to locate our meeting point (Visalli \& Vallesi, 2018; Vallesi, 2014), or when we predict the timing at which the yellow traffic light will turn red (Nobre et al., 2007; Vallesi et al., 2013). These real-life examples show that monitoring abilities may be operationalized in laboratory settings either as sustained processes operating throughout the trial or as more transient processes acting on each individually presented item (Braver et al., 2003; Tarantino et al., 2017).

The variable foreperiod task is a widely used experimental paradigm to investigating sustained monitoring processes. In such a task, participants respond to the onset of a target appearing with the same a priori probability after variable foreperiod durations (i.e., time interval between warning and 
target stimuli). Participants are usually faster in responding to targets appearing after longer foreperiods compared with shorter ones, provided that foreperiods are varied randomly and equiprobably (i.e., "the variable foreperiod effect"; see Capizzi \& Correa, 2018; Coull, 2009; Niemi \& Näätänen, 1981; Vallesi, 2010, for reviews). Indeed, supposing that there are only two possible foreperiods, once the short foreperiod has passed by, the probability that the target will occur at the long foreperiod is equal to $100 \%$. Implicitly monitoring the conditional probabilities of target onset during the trial will, thus, foster better performance at the long foreperiod (Steinborn \& Langner, 2012; Vallesi \& Shallice, 2007). Accordingly, the monitoring ability may be operationalized as the Response Time (RT) difference between short and long foreperiod trials.

An example of transient monitoring can be found in the study by Capizzi et al. (2016). They asked participants to monitor whether a three-dimensional word contained a certain consonant or displayed a specific spatial configuration. The nature of these monitoring tasks hence required a more transient event-related activity to check whether each word had a target status or not. As a baseline (nonmonitoring) condition, in separate block of trials participants classified the words without extra monitoring requirements. Therefore, monitoring in these target-checking tasks may be operationalized as the RT difference between trials in monitoring blocks and trials in nonmonitoring blocks. We will turn back to these behavioral indices in the Methods section.

Despite the monitoring processes assessed by foreperiod and target-checking paradigms have different temporal profiles (i.e., sustained vs. transient), they all have been related to the activity of the right prefrontal cortex (PFC) and associated neural networks (Capizzi et al., 2016; Coull et al., 2000; Vallesi et al., 2009). Converging evidence from both neuropsychology and functional magnetic resonance imaging (fMRI) further strengthens the hypothesis that monitoring processes are (mainly) right-lateralized in the PFC (see Vallesi, 2012, for an overview). This right hemispheric specialization also is believed to be independent of the specific (possibly lateralized) task domains employed, such as the spatial and verbal ones (Capizzi et al., 2016), or the verbal episodic memory retrieval and perceptual decision making (Fleck et al., 2006).

In the present study, we went a step further by investigating whether intrinsic (i.e., resting-state-related) right-lateralized brain dynamics also may contribute explaining interindividual variability in monitoring, independently of both the specific task cognitive domain and temporal characteristics of the involved monitoring processes. Our starting point was the mounting body of evidence from neuroimaging studies showing that the pattern of spontaneous fluctuations in cerebral activity can explain task-evoked brain responses and behavioral performance in several task domains, such as working memory (Zou et al., 2013; see also van Dam et al., 2015), object color knowledge (Wang et al., 2013), interference resistance (Coste et al., 2011), attention (Xu et al., 2014; Fryer et al., 2015), unsupervised learning (Wong et al., 2014), word reading (Xu et al., 2015), and verbal fluency (Yin et al., 2015). Along the same lines, Ambrosini and Vallesi (2016, 2017) recently showed that differences in resting-state brain dynamics, as assessed by source-based EEG spectral analysis and related hemispheric asymmetries, partly explained individual differences in criterion-setting, an executive function reflecting the ability to flexibly set up and select task rules. This function requires the causal involvement of the left prefrontal cortex (Jahanshahi et al., 1998; Shallice et al., 2007, 2008; Stuss, 2011; Stuss and Alexander, 2007; Vallesi, 2012). As behavioral indices of criterion-setting, both switching costs (Ambrosini \& Vallesi, 2016) and Stroop effects (Ambrosini \& Vallesi, 2017) were calculated in two separate studies. Overall, the results showed that participants with left-lateralized intrinsic brain activity were more able to rapidly switch between different tasks and to resolve Stroop interference. Given that such findings were found using both verbal and nonverbal tasks, it was then concluded that the observed left hemispheric asymmetries were driven by the criterion-setting process and not by the specifics of the to be performed tasks. Moreover, as detailed below, unlike criterion-setting abilities indexed by switching costs, monitoring abilities measured through mixing costs were instead associated with right prefrontal brain activity at rest (Ambrosini and Vallesi, 2016).

In summary, spontaneous brain dynamics may provide a prior for task-related activity, intended as a functional bias, or even "the entire set of individual inner cortical and cognitive biases" (Harmelech \& Malach, 2013, p. 607; see also Fiser et al., 2010; Sadaghiani \& Kleinschmidt, 2013). Following this line of reasoning, and considering the important role played by the right (prefrontal nodes of the) fronto-parietal brain network in mediating monitoring processes, we asked whether participants with stronger right-lateralized intrinsic brain activity would be those better able to accomplish the monitoring task requirements. To do this, we related the above-mentioned behavioral indices obtained in the foreperiod, verbal, and spatial target-checking tasks to lateralized electroencephalographic (EEG) brain activity at rest. As a measure of brain dynamics at rest, we used the ratio between the power in beta and alpha frequency bands. Such an index was preferred over other measures, because it has been shown to reflect intrinsic electrophysiological dynamics related to increased attentional investment and cortical engagement in information processing (Laufs et al., 2006), which is of particular importance to our aim (see the "Resting State EEG Recording and Analysis" section for further details). Moreover, the implementation of the same $\beta / \alpha$ ratio measure as that used in our previous two studies (Ambrosini \& Vallesi, 2016, 2017) allowed us to make a more direct comparison between our prior (leftlateralized) criterion-setting results and the present (plausible right-lateralized) monitoring ones. 


\section{Materials and methods}

\section{Participants}

Fifty-six university students participated in the experiment after providing informed consent. These participants were the same as in our previous studies (Ambrosini \& Vallesi, 2016, 2017). Two participants were excluded from the analysis because of low accuracy $(\leq 50 \%)$ in the Target condition of the verbal ( 1 participant) and spatial (1 participant) monitoring tasks (see "Verbal and spatial event-related monitoring tasks" section). All participants reported no history of neurological or psychiatric disorders, had normal color vision and normal or corrected-to-normal visual acuity, and were right-handed according to the Edinburgh Handedness Inventory (Oldfield, 1971) with an average score of 80.3 (standard deviation $[\mathrm{SD}]=16.5)$. All of them signed a written, informed consent before the experiment and were reimbursed for their time. The study was approved by the Bioethical Committee of the Azienda Ospedaliera di Padova and was conducted according to the guidelines of the Declaration of Helsinki (World Medical Association, 2013).

\section{Behavioral tasks}

For all our tasks, stimulus presentation and data recording were controlled by E-prime 2 software (Schneider et al., 2002). Figure 1 summarizes our monitoring tasks.

\section{Foreperiod task}

The Foreperiod (FP) dual-task paradigm was adapted from Vallesi et al. (2014). A first block of pure FP trials (4 practice trials and 30 test trials) was used to calculate the FP effect without dual-task demands. During this first block, each trial started with the presentation of a yellow "XX" symbol, which was displayed in the center of the screen simultaneously with an auditory warning signal (1,500-Hz pure tone). The warning signal was played for $50 \mathrm{~ms}$ via laptop internal speakers at a sound intensity level comfortable for all the participants. The "XX" symbol was then replaced by the target (a downwardpointing white arrow, with the maximum length and width of $2 \mathrm{~cm}$ ) at a variable foreperiod of either 3,000 or $5,000 \mathrm{~ms}$. Participants had to respond to the target by pressing the spacebar as quickly as possible. Following the response to the target, or after 2,000 $\mathrm{ms}$ in case of a missed response, the next trial began. A second block of FP trials under dual-task condition (4 practice trials and 60 test trials) was then administered, but the data from this block were discarded from the present analyses, because they were not relevant for our hypotheses.
Verbal and spatial event-related monitoring tasks

The procedure and the monitoring tasks are the same as in our previous study (Capizzi et al., 2016). Briefly, stimulus material consisted of three-dimensional visually presented words that could be categorized within verbal or spatial contexts. The verbal context comprised two subtasks that had to be performed one at a time. In the "gender" subtask, participants discriminated between female and male names, whereas in the "name" subtask the choice regarded the proper or common name status of the word. In the spatial context, participants had to categorize the words according to their "roll" (i.e., clockwise vs. counterclockwise) or "pitch" (i.e., upward vs. downward) rotation. The word colors changed randomly between green and brown in every trial, although this colorchange was task-irrelevant and introduced only to match this low-level feature of the present task with that of the taskswitching paradigm (see Ambrosini \& Vallesi, 2017). Verbal and spatial task sessions were blocked and administered in a counterbalanced order across participants, as was counterbalanced the assignment of categories to response keys (i.e., the " $\mathrm{f}$ " key and the " $\mathrm{k}$ " key on the computer keyboard).

For both verbal and spatial tasks, trials comprised $2,000 \mathrm{~ms}$ of word presentation and $1,800 \mathrm{~ms}$ of intertrial interval. A total of 2 nonmonitoring blocks, each comprising 32 trials, and 2 monitoring blocks, each comprising 32 trials of which 4 or 5 were target trials, were presented for both the verbal and the spatial sessions. In the verbal monitoring blocks, participants had to monitor whether the word contained the consonant "v." Whenever this happened, they had to refrain from implementing the gender or name subtask rules and press the spacebar with the thumb of their right hand. In the spatial monitoring blocks, the task was to monitor for the word rotated to $45^{\circ}$ in either a clockwise or counterclockwise direction, with similar task requirements as for the verbal version.

The experimental blocks were preceded by a short practice session that allowed participants to familiarize themselves with the appropriate task stimulus-response mappings. Both the gender and the name subtasks (for the verbal domain) and the roll and the pitch ones (for the spatial domain) were collapsed in order to increase the signal-to-noise ratio of EEG data. Moreover, the infrequent target trials were discarded from all the analyses.

\section{Behavioral data analysis}

Error trials, anticipations (RTs $<100 \mathrm{~ms}$ ), and trials with missed responses were discarded from the analyses (1.79\%, $3.80 \%$, and $5.93 \%$ for the FP, verbal, and spatial monitoring tasks, respectively). The RTs were log transformed (natural base) to improve normality. Moreover, to obtain central tendency measures that were as robust as possible against the influence of outliers, a robust M-estimator of location 


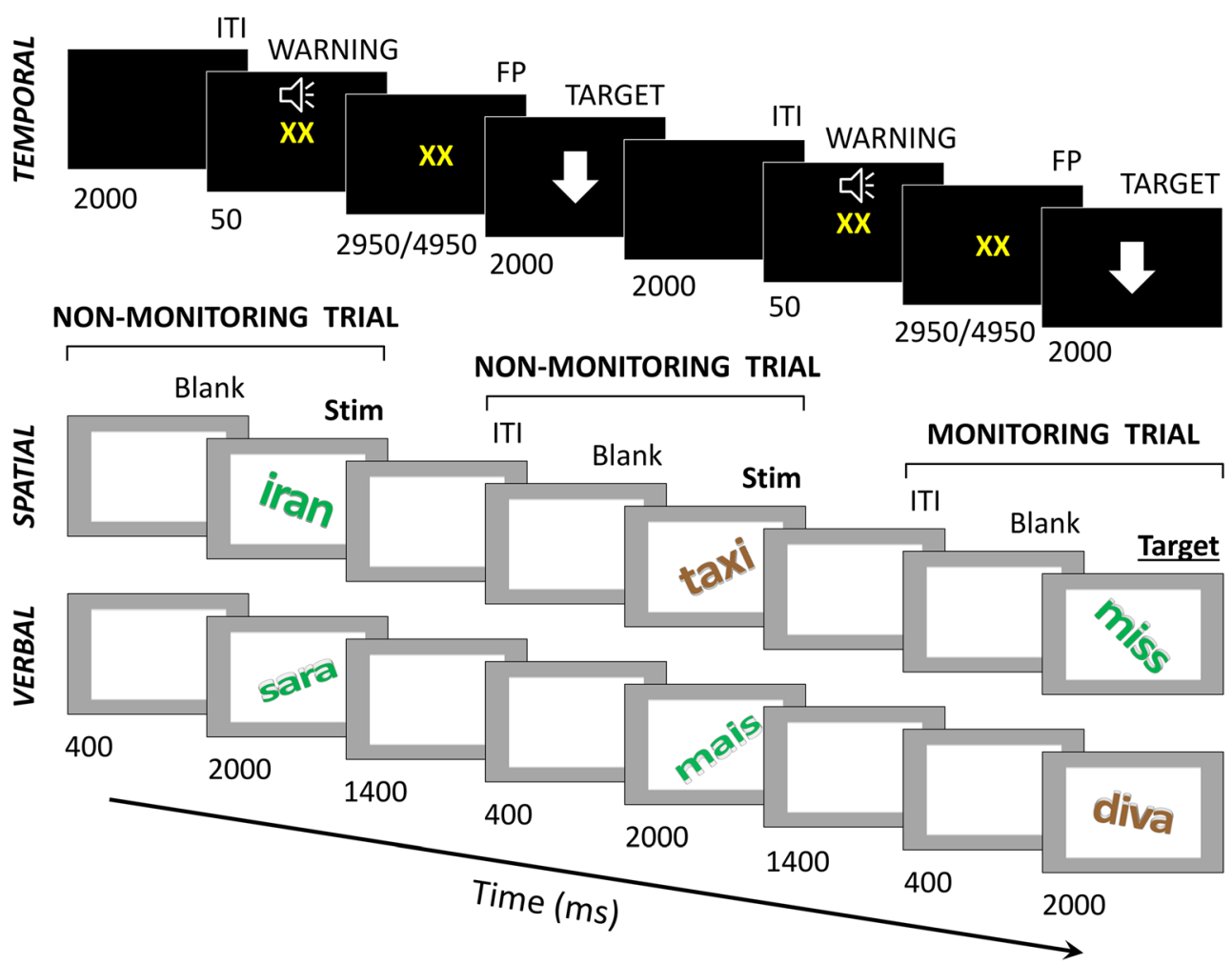

Fig. 1. Experimental tasks. The trial structure of the temporal (foreperiod), spatial, and verbal versions of the monitoring task is shown (from top to bottom). Three trials are shown for the foreperiod

(robust mean RTs; for details, see Ambrosini \& Vallesi, 2016; see also Rousseeuw \& Verboven, 2002) was computed for each participant and condition separately using the LIBRA MATLAB library (Verboven \& Hubert, 2005, 2010). After these transformations, the variables showed acceptable skewness and kurtosis (all $<|0.63|)$.

The FP effect was calculated as the difference between the robust mean RTs of short and long FP conditions. This index represents the participants' temporal monitoring ability, that is, their ability to optimize response preparation by monitoring the time-dependent changes in the conditional probability of stimulus occurrence during the foreperiod. The monitoring effects for the verbal and spatial tasks were computed as the difference between the robust mean RTs from the nonmonitoring blocks and that from nontarget trials of the monitoring blocks. These indices represent the performance cost (i.e., a lengthening of RTs) in performing the verbal and spatial tasks, which was caused by the monitoring processes occurring on a trial-by-trial basis to check for target presence. As such, these monitoring effects quantify a monitoring-dependent cost in performance, rather than an advantage as in the case of the temporal monitoring effect described above for the FP task. For this reason, the verbal and spatial monitoring effects were inverted, so that all the three effects represented monitoring ability rather than a monitoring deficit. task. A monitoring and two nonmonitoring trials are shown for the spatial and verbal event-related monitoring tasks. See Sections 2.2.1 and 2.2.2 for more details.

The statistical significance of the three monitoring effects was assessed by means of one-sample $t$-tests against zero. The Cohen's $d$ was used as the measure of the effect size (Cohen, 1977). The monitoring effects were $z$-transformed before proceeding to the EEG spectral power-behavior correlation analyses to allow comparison of the results across different cognitive domains.

\section{Resting state EEG recording and analysis}

A single, 5-min eye-closed resting state EEG (rsEEG) session was recorded with a 64-channels system (BrainProducts, Munich, Germany; sampling rate $=500 \mathrm{~Hz}$; online bandpass filter $=0.1-100 \mathrm{~Hz}$; impedances $<10 \mathrm{k} \Omega$ ). Electrooculographic activity also was recorded with an electrode placed under the left eye. The electrodes located at $\mathrm{FCz}$ and $\mathrm{AFz}$ served as the online reference and ground, respectively.

Offline rsEEG processing and analyses, distributed source imaging, and spectral power analysis of the estimated cortical sources activity were performed as detailed in Ambrosini and Vallesi (2017). We performed source analyses to derive our laterality indices as the effects might not be generalized to scalp-based laterality measures (Gordon et al., 2018). We shall briefly describe these procedures in what follows. 
The offline EEG preprocessing was performed by using custom MATLAB (The MathWorks, Inc., Natick, MA) scripts based on functions from the EEGLAB environment (version 12.0.2b; Delorme and Makeig, 2004). After band-pass filtering $(0.5-45 \mathrm{~Hz})$ of continuous EEG data, we performed an automatic channel rejection procedure and confirmed its outcome by visual inspection. Mastoid channels (TP9 and TP10) were excluded from the analyses due to excessive noise and other rejected channels were interpolated using spherical splines (Perrin et al., 1989). Then, EEG data were segmented into 2048-ms epochs, re-referenced to the common average reference, and submitted to an automatic epoch rejection procedure based on extreme values, linear trend, improbability, kurtosis, and spectral profile tests (Delorme, Sejnowski, \& Makeig, 2007; for details, see Ambrosini \& Vallesi, 2016).

Next, the source imaging of the artifact-free rsEEG epochs was performed with Brainstorm (Tadel et al., 2011) using a 15002-dipoles distributed source model (Colin27, derived from the standard $1 \mathrm{~mm}$ resolution brain of the Montreal Neurological Institute), a forward model generated with OpenMEEG using a three-layer symmetric boundary element method (Gramfort et al., 2010; Kybic et al., 2005), and a depth-weighted minimum-norm estimation algorithm (Baillet et al., 2001). The cortical surface was parcellated into 150 anatomical regions derived from a modified version of the Destrieux atlas (Destrieux et al., 2010), which ensured a more homogeneous covering of the cortical surface with a smaller across-regions variability in number of vertices as compared to the original atlas (Ambrosini \& Vallesi, 2016).

We then computed the power spectral densities (PSDs) of the electrical activity of the 15002 sources using the EEGLAB (Delorme \& Makeig, 2004) spectopo function (frequency range $=1-45 \mathrm{~Hz}$; resolution $=0.25 \mathrm{~Hz}$ ) and averaged the obtained PSDs over the vertices composing each region. Finally, hemispheric asymmetry in intrinsic brain dynamics for each pair of regions was operationalized by computing the $\beta / \alpha$ hemispheric asymmetry score $(\beta / \alpha$ HAS $)$, that is, the right-left difference in the log-transformed ratio between power in $\beta(12.5-24 \mathrm{~Hz})$ and $\alpha(7.5-12.5 \mathrm{~Hz})$ bands. We chose to use the $\beta / \alpha$ ratio, instead of the relative power in a specific frequency band, because the latter approach can overlook the complexity of the EEG spectrum structure, as well as the fact that particular neural processes may be reflected in several frequency bands concurrently. In general, to date there is a consensus that the activity in distinct brain networks may correlate with the power in multiple frequency bands and, conversely, distinct frequency bands may correlate with multiple fMRI networks (Mantini et al., 2007; Laufs, 2008). Moreover, previous studies using combined electrical (i.e., EEG-derived) and hemodynamic (i.e., BOLD) measurements of brain activity suggested that changes in BOLD are associated with changes in the spectral profile of neuronal activity that do not arise from one specific frequency band but rather from the relative power between high and low frequencies, with an overall shift in the frequency center toward higher frequencies (Kilner et al., 2005; Rosa et al., 2010) and, thus, an increase in $\beta / \alpha$. The $\beta / \alpha$ ratio would thus represent a better quantitative measure of intrinsic (i.e., resting-state-related) brain activity compared with the power in specific frequency bands (for a detailed discussion, see Ambrosini \& Vallesi, 2016). More importantly, the $\beta / \alpha$ ratio would better reflect resting-state-related attentional investment and neural engagement in information processing, with a high-arousal state and an "attentional" parieto-frontal pattern of fMRI activations (Laufs et al., 2006; Ota et al., 1996).

We also performed additional control analyses to assess the functional specificity of the $\beta / \alpha$ HAS measure. To this aim, we investigated the relation between participants' monitoring ability and two other rsEEG asymmetry scores we investigated in our previous studies (Ambrosini \& Vallesi, 2016, 2017), that is, the $\beta / \theta_{-}$HAS and $\gamma / \alpha_{-}$HAS. These measures were computed as done for the $\beta / \alpha$ HAS (see above) as the right-left difference in the ratio between power in beta and theta $(\theta, 4-7.5 \mathrm{~Hz})$ bands and that between power in gamma $(\gamma, 24-45 \mathrm{~Hz})$ and alpha frequency bands, respectively. Finally, to facilitate the functional interpretation of the $\beta / \alpha$ _HAS results and to investigate whether they could have been driven by single frequency bands, we also investigated the power-behavior correlations in the right-left hemispheric asymmetry score in the relative power in the $\alpha$ and $\beta$ frequency bands ( $\alpha \_$HAS and $\beta$ HAS, respectively).

\section{Inferential statistics}

We performed a series of robust correlation analyses (i.e., skipped Pearson correlations; Wilcox, 2004) between the $\beta / \alpha$ _HAS values for each region, on the one side, and the temporal, verbal and spatial $z$-transformed monitoring effects, on the other side. We chose to use robust correlations instead of regular ones because they ensure an accurate control of the false positive rate and provide an adequate estimate of the true correlation in the data without substantial losses of power when data contain outliers, especially bivariate ones (Ambrosini \& Vallesi, 2017). Statistical significance testing for the predicted positive correlations between $\beta / \alpha$ HAS and monitoring ability measures was conducted using the bootstrap test (2000 resamples; one-sided $95 \%$ confidence intervals: $B-C I_{95}$ ), which is more robust against heteroscedasticity compared with traditional $t$-tests (Pernet et al., 2012).

Moreover, because our goal was to test whether the hemispheric asymmetry in intrinsic brain dynamics was positively related to monitoring ability regardless of the cognitive domain, we performed an intersection analysis to identify the regions for which significant skipped correlations were found between the $\beta / \alpha$ HAS and all the three monitoring ability 
measures. This also helped us to better control the inflation of Type-I error rate due to multiple comparisons. The results of the intersection analysis were corrected for multiple comparisons by controlling for the Family-wise error (FWE) rate using Monte Carlo simulations based on the inter-correlation structure of our behavioral and electrophysiological data. Similar intersection analyses were performed for the control analyses on the skipped correlations between the other spectral measures and the three monitoring ability measures.

We also compared the three monitoring ability measures and computed the skipped correlations between them to assess the similarity between the participants' monitoring ability across cognitive domains. Moreover, to evaluate the relationship between the participants' monitoring ability investigated here and their criterion-setting and monitoring abilities investigated in our previous individual difference companion studies, we also computed the skipped correlations between, on the one side, the three monitoring ability measures used here and, on the other side, both the general mixing costs assessing monitoring ability (Ambrosini \& Vallesi, 2016) and the general switching costs and the verbal and spatial Stroop effects assessing criterionsetting ability (Ambrosini \& Vallesi, 2016, 2017).

Finally, to clarify the functional meaning of the powerbehavior correlations reported for the mMFG cortical region and to investigate whether there is some underlying asymmetric process that is related to both criterion-setting and monitoring processes, we performed a differential correlation analysis by comparing the correlations between the $\beta / \alpha \_$HAS and all the three monitoring ability measures reported and 1) the correlation between the $\beta / \alpha \_$HAS and both the general mixing costs assessing monitoring ability and 2) the general switching costs assessing criterion-setting ability, which we reported in our first companion paper (Ambrosini \& Vallesi, 2016), and 3) the correlation between the $\beta / \alpha \_$HAS and the spatial and verbal Stroop effects assessing criterion-setting ability, which we reported in our second companion paper (Ambrosini \& Vallesi, 2017). In the first two cases, we performed the Zou's confidence interval (Zou, 2007) and the Steiger's $z$ tests (Steiger, 1980) for comparing dependent, nonoverlapping correlations while accounting for the pattern of intercorrelations between all the variables entered into the analysis, as the $\beta / \alpha$ HAS measure was computed with a slightly different approach in our first companion paper (Ambrosini \& Vallesi, 2016). In the third case, we used the same tests for comparing dependent, overlapping correlations while accounting for the correlation between the behavioral measures, as the $\beta / \alpha$ HAS measure was computed with the same approach used here (Ambrosini \& Vallesi, 2017). In all cases, a casewise deletion of missing/outlier data was employed. Bootstrap-based $p$ values were corrected for multiple comparisons using the false discovery rate method at a 0.05 level (Benjamini and Hochberg, 1995)

\section{Results}

\section{Behavioral results}

The behavioral analysis for the foreperiod task revealed a significant temporal monitoring effect $(M=0.146, S D=$ $0.082, t_{(53)}=13.03, p<10^{-17}, d=1.77$; Fig. 2 ), with longer RTs for the short FP condition as compared to the long one (respectively, $M=5.80$ and $5.66, S D=$ 0.14 and 0.15 ). This temporal monitoring effect was exhibited by all the participants with a substantial interindividual variability (Fig. 2).

The behavioral analysis for the verbal and spatial eventrelated monitoring tasks revealed significant monitoring effects in both tasks (respectively, $M=0.131$ and $0.274, S D=$ 0.111 and $0.124, t_{(53)}=8.67$ and $16.27, p<10^{-11}$ and $10^{-21}, d=$ 1.18 and 2.21; Fig. 2), with longer RTs for the monitoring condition (respectively, $M=6.70$ and $6.78, S D=0.16$ and 0.18 ) compared with the nonmonitoring one (respectively, $M$ $=6.56$ and $6.51, S D=0.15$ and 0.16 ). These monitoring effects were exhibited by the vast majority of participants (respectively, 53/54 and 47/54 for the verbal and spatial task) with a substantial inter-individual variability that was similar between the two tasks $\left(F_{(53,53)}=1.23, p=0.449, F\right.$ test for equality of variances) and higher compared with that observed for the temporal monitoring task (respectively, $F_{(53,53)}=1.84$ and 2.27, $p=0.028$ and 0.003; Fig. 2).

The spatial monitoring effect was significantly higher than both the verbal $\left(t_{(53)}=7.104, p>10^{-8}, d=0.967\right)$ and the temporal $\left(t_{(53)}=6.406, p>10^{-7}, d=0.872\right)$ ones, which did not differ between each other $\left(t_{(53)}=0.895, p=0.375, d=\right.$ $0.122)$. Finally, while there was a significant correlation between the verbal and spatial monitoring effects (skipped $r=$ $\left.0.385, B-C I_{95 \%}=[0.127,0.614]\right)$, these effects were not correlated to the temporal monitoring one (respectively, skipped $r$ $=0.137$ and $0.017, B-C I_{95 \%}=[-0.124,0.398]$ and $[-0.281$, $0.389]$ ), thus suggesting the existence not only of domain independence, but also of some degree of domain specificity in monitoring processes.

As regards the comparison with our previous studies, were significantly correlated to the general mixing costs (respectively, skipped $r=0.378$ and $0.365, B-C I_{95 \%}=[0.135$, $0.573]$ and $[0.134,0.556])$, but the same was not true for the temporal monitoring effect (skipped $r=-0.1813, B-C I_{95 \%}=$ $[-0.463,0.124])$. The spatial monitoring effect also showed a significant negative correlation with the general switching cost (skipped $\left.r=-0.408, B-C I_{95 \%}=[-0.592,0.191]\right)$. None of the other correlations was significant (all uncorrected $p \mathrm{~s}>$ 0.084). These results indicate the existence of some degree of association between the different cognitive control processes supposed to be mediated by the monitoring executive function; they also suggest the independence between these monitoring-related processes and those supposed to be 


\section{Temporal}

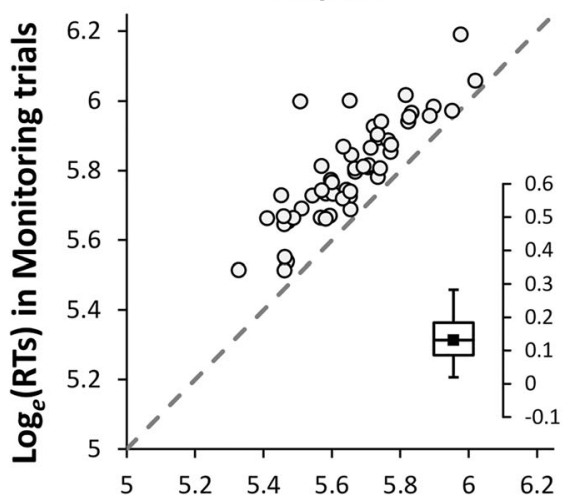

Verbal

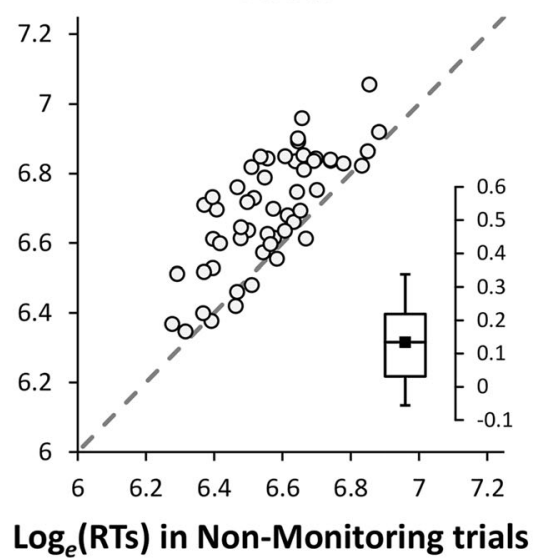

Spatial

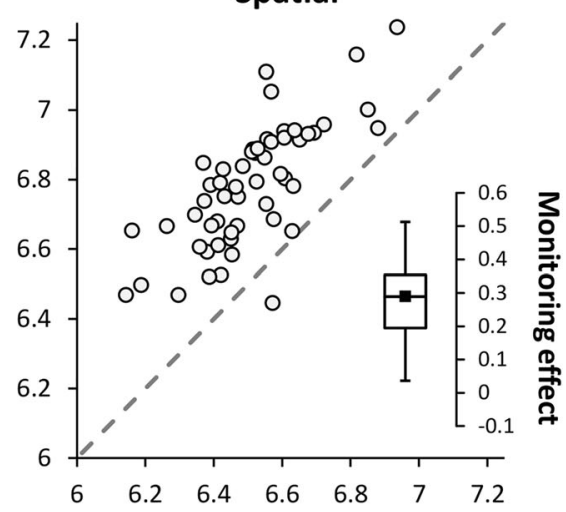

Fig. 2. Participants' behavioral performance in the monitoring tasks. Scatterplots showing the participants' mean M-estimates of natural logtransformed RTs in nonmonitoring ( $x$ axis) and monitoring ( $y$ axis) trials for the temporal (left), verbal (middle), and spatial (right) monitoring tasks. Circles represent data from each participant. The diagonal dashed lines indicate the identity line $(y=x)$, so that circles above the diagonal

mediated by the criterion-setting executive function (i.e., those assessed by the switching and Stroop effects).

\section{Power-behavior correlations}

A number of regions showed significant correlation between $\beta / \alpha \_$HAS and each of the three measures of monitoring ability, as detailed in Table 1 (Fig. 3). Many of these regions were not overlapping across the three measures. This again indicates that some degree of domain specificity mediates the relationship between participants' hemispheric asymmetry in intrinsic electrophysiological activity and their differences in monitoring abilities. However, we specifically aimed to identify the cortical regions where the rsEEG power-behavior correlation was significant regardless of the cognitive domain and specific characteristics of the tasks employed. Therefore, domain-specific effects fall beyond the scope of this study and will not be discussed further.

The results of the subsequent intersection analysis revealed that five cortical regions showed a right lateralization of intrinsic brain dynamics that was significantly related to each and every measure of monitoring ability (Table 2; Fig. 3 ). Interestingly, one of these was the middle portion of the middle frontal gyrus (mMFG). This was the same prefrontal ROI whose hemispheric asymmetry in rsEEG spectral activity was found to be significantly and oppositely related to behavioral measures of phasic cognitive control abilities related to criterion-setting processes and, importantly, a behavioral measure of cognitive control ability related to monitoring processes (Ambrosini \& Vallesi, 2016, 2017). The intersection analysis also revealed two other prefrontal regions, one located ventrally to the mMFG, that is, the inferior frontal sulcus (IFS), and the more rostral prefrontal cortex represent participants exhibiting a raw monitoring effect. The boxplots in the insets show the distribution of the participants' monitoring effects; the central line/point of the box represents the median, the edges of the box are the first and third quartiles, and the whiskers represent the range of the data.

(anterior prefrontal cortex, aPFC), albeit the results for this latter region did not survive the correction for multiple comparisons. The two remaining regions were located in the medial wall of the brain: the dorsal anterior cingulate cortex (dACC) in the frontal lobe, and the posterior cingulate (marginal) sulcus (pCingS) in the parietal lobe. For all these regions, participants with a right-lateralized rsEEG spectral activity (i.e., showing higher $\beta / \alpha$ power in the right than left hemisphere) had significantly better monitoring abilities regardless of the cognitive domain and task characteristics (Fig. 3), i.e., they were more able to exert cognitive control to monitor for relevant information and to optimize their behavior.

We also performed a differential correlation analysis in order to clarify the functional meaning of the powerbehavior correlations reported here for the mMFG. This analysis revealed that the relationship between monitoring ability and right hemispheric asymmetry in mMFG intrinsic spectral activity reported in the present study was not statistically different from the one observed in our previous study for the monitoring-related general mixing costs (Ambrosini \& Vallesi, 2017), as none of the tests comparing these correlations was statistically significant (verbal monitoring: $z=$ $0.796, p=0.426, \mathrm{CI}=[-0.159,0.375]$; spatial monitoring: $z$ $=0.718, p=0.472, \mathrm{CI}=[-0.200,0.429]$; temporal monitoring: $z=0.253, p=0.800, \mathrm{CI}=[-0.438,0.339])$. On the contrary, the differential correlation analysis revealed that the hemispheric asymmetry in mMFG intrinsic spectral activity reported in the present study was significantly correlated in an opposite way to the monitoring-related behavioral measures reported here and the criterion-setting-related behavioral measures reported in our previous studies (Ambrosini \& Vallesi, 2016, 2017). Indeed, the participants' mMFG $\beta / \alpha$ HAS 
Table 1. Results of the robust correlation analysis. The table reports the cortical regions that showed significant correlations between EEG asymmetries and performance for up to two monitoring tasks

\begin{tabular}{|c|c|c|c|c|c|c|c|c|c|}
\hline \multirow[t]{2}{*}{ Region } & \multicolumn{3}{|c|}{ Temporal monitoring } & \multicolumn{3}{|c|}{ Verbal monitoring } & \multicolumn{3}{|c|}{ Spatial monitoring } \\
\hline & $r_{s k i}$ & ${ }_{L B} C I_{95 \%}$ & ${ }_{U B} C I_{95 \%}$ & $r_{s k i}$ & ${ }_{L B} C I_{95 \%}$ & ${ }_{U B} C I_{95 \%}$ & $r_{s k i}$ & ${ }_{L B} C I_{95 \%}$ & ${ }_{U B} C I_{95 \%}$ \\
\hline pSPL & -0.237 & -0.474 & -0.010 & -0.228 & -0.456 & -0.011 & 0.100 & -0.097 & 0.287 \\
\hline MFS & 0.010 & -0.213 & 0.225 & -0.215 & -0.423 & -0.001 & -0.266 & -0.499 & -0.014 \\
\hline pMFG & -0.079 & -0.328 & 0.168 & -0.242 & -0.441 & -0.014 & -0.235 & -0.477 & -0.002 \\
\hline IFGorb & 0.188 & -0.079 & 0.438 & -0.307 & -0.524 & -0.037 & -0.368 & -0.580 & -0.098 \\
\hline iPreCS & -0.067 & -0.278 & 0.166 & -0.326 & -0.534 & -0.075 & -0.293 & -0.506 & -0.042 \\
\hline OrG & -0.019 & -0.266 & 0.199 & -0.326 & -0.537 & -0.097 & -0.292 & -0.528 & -0.060 \\
\hline vLS & 0.036 & -0.223 & 0.293 & -0.257 & -0.506 & -0.003 & -0.268 & -0.472 & -0.028 \\
\hline aSPL & -0.291 & -0.515 & -0.015 & -0.137 & -0.397 & 0.145 & -0.091 & -0.339 & 0.163 \\
\hline POS & -0.288 & -0.506 & -0.013 & -0.169 & -0.427 & 0.133 & 0.100 & -0.142 & 0.319 \\
\hline PreCun & -0.263 & -0.475 & -0.013 & 0.041 & -0.383 & 0.195 & 0.060 & -0.189 & 0.297 \\
\hline aMTG & 0.190 & -0.068 & 0.414 & -0.395 & -0.605 & -0.128 & -0.209 & -0.458 & 0.060 \\
\hline LOTS & -0.003 & -0.264 & 0.255 & -0.354 & -0.570 & -0.088 & -0.209 & -0.461 & 0.042 \\
\hline SFS & -0.106 & -0.338 & 0.126 & -0.341 & -0.529 & -0.115 & -0.119 & -0.378 & 0.154 \\
\hline FusG & -0.057 & -0.307 & 0.201 & -0.330 & -0.529 & -0.077 & -0.084 & -0.325 & 0.155 \\
\hline Tpole & -0.041 & -0.291 & 0.202 & -0.290 & -0.509 & -0.049 & -0.205 & -0.433 & 0.037 \\
\hline OrS & 0.125 & -0.123 & 0.373 & -0.282 & -0.504 & -0.014 & -0.131 & -0.343 & 0.096 \\
\hline pSFG & -0.048 & -0.280 & 0.184 & -0.114 & -0.348 & 0.137 & -0.314 & -0.503 & -0.089 \\
\hline sInsG & 0.165 & -0.086 & 0.421 & -0.195 & -0.445 & 0.097 & -0.301 & -0.527 & -0.016 \\
\hline CircInsS & 0.108 & -0.105 & 0.320 & -0.173 & -0.417 & 0.111 & -0.281 & -0.521 & -0.022 \\
\hline $\mathrm{mpSFG}$ & -0.138 & -0.378 & 0.120 & -0.170 & -0.416 & 0.123 & -0.260 & -0.513 & -0.003 \\
\hline
\end{tabular}

Note: $r_{\text {ski }}$, skipped Pearson's correlation; ${ }_{L B} C I_{95}$ and ${ }_{U B} C I_{95} \%$, lower and upper bound of the $95 \%$ bootstrap confidence interval, respectively. Bold indicates correlations that were significant at the nonparametric percentile bootstrap test (see Section 2.5). aMTG, anterior part of the middle temporal gyrus; aSPL, anterior part of the superior parietal lobule; CircInsS, circular insular sulcus; FusG, fusiform gyrus; IFGorb, orbital inferior frontal gyrus; iPreCS, inferior part of the precentral sulcus; LOTS, lateral occipito-temporal sulcus; MFS, middle frontal sulcus; mpSFG, mid-posterior part of the superior frontal gyrus; OrG, orbital gyrus; OrS, orbital sulcus; pMFG, posterior part of the middle frontal gyrus; POS, parieto-occipital sulcus; PreCUN, precuneus; pSFG, posterior part of the superior frontal gyrus; pSPL, posterior part of the superior parietal lobule; SFS, superior frontal sulcus; sInsG, short insular gyrus; Tpole, temporal pole; vLS, vertical ramus of the lateral sulcus.

values were positively correlated with their monitoring ability scores and negatively correlated with the behavioral scores assessing both their general switching ability (for the verbal, spatial, and temporal monitoring scores, respectively, $z=$ $3.614,3.249$, and 2.584; $p=0.0003,0.001$, and 0.010 ; $\mathrm{CI}=$ $[0.300,1],[0.238,1]$, and $[0.119,0.898])$ and their verbal (respectively, $z=2.705,2.526$, and 2.146; $p=0.003,0.006$, and 0.016; $\mathrm{CI}=[0.174,0.958],[0.133,0.929]$, and [0.046, $0.870]$ ) and spatial (respectively, $z=3.391,3.348$, and 2.912; $p=0.0003,0.0004$, and $0.002 ; \mathrm{CI}=[0.305,1],[0.324,1]$, and $[0.215,0.969]))$ Stroop interference resistance ability, which all are supposed to be mediated by the criterion-setting executive function (Ambrosini et al., 2019).

These results thus indicate the existence of a bivalent association between the $\beta / \alpha$ HAS at rest in the mMFG and both the monitoring and criterion-setting control processes: Participants with a more right-lateralized intrinsic brain activity in the mMFG had better monitoring-related control abilities, whereas participants with a more left-lateralized intrinsic brain activity in the same PFC region had better criterionsetting-related control abilities.

Regarding the intersection analyses on the other spectral measures, none of them showed significant domainindependent power-behavior correlations in any cortical region after controlling for Type-I error inflation due to multiple comparisons. Indeed, only two cortical regions (including the $\mathrm{mMFG}$ ) were significant at the intersection analysis, showing significant correlations between all of the three monitoring ability scores and one of the spectral measures, the $\beta$ HAS. However, these results did not survive the correction for multiple comparisons.

\section{Discussion}

Two previous individual difference companion studies on the relationship between executive functions and resting-state electrophysiological brain dynamics have shown that the huge 

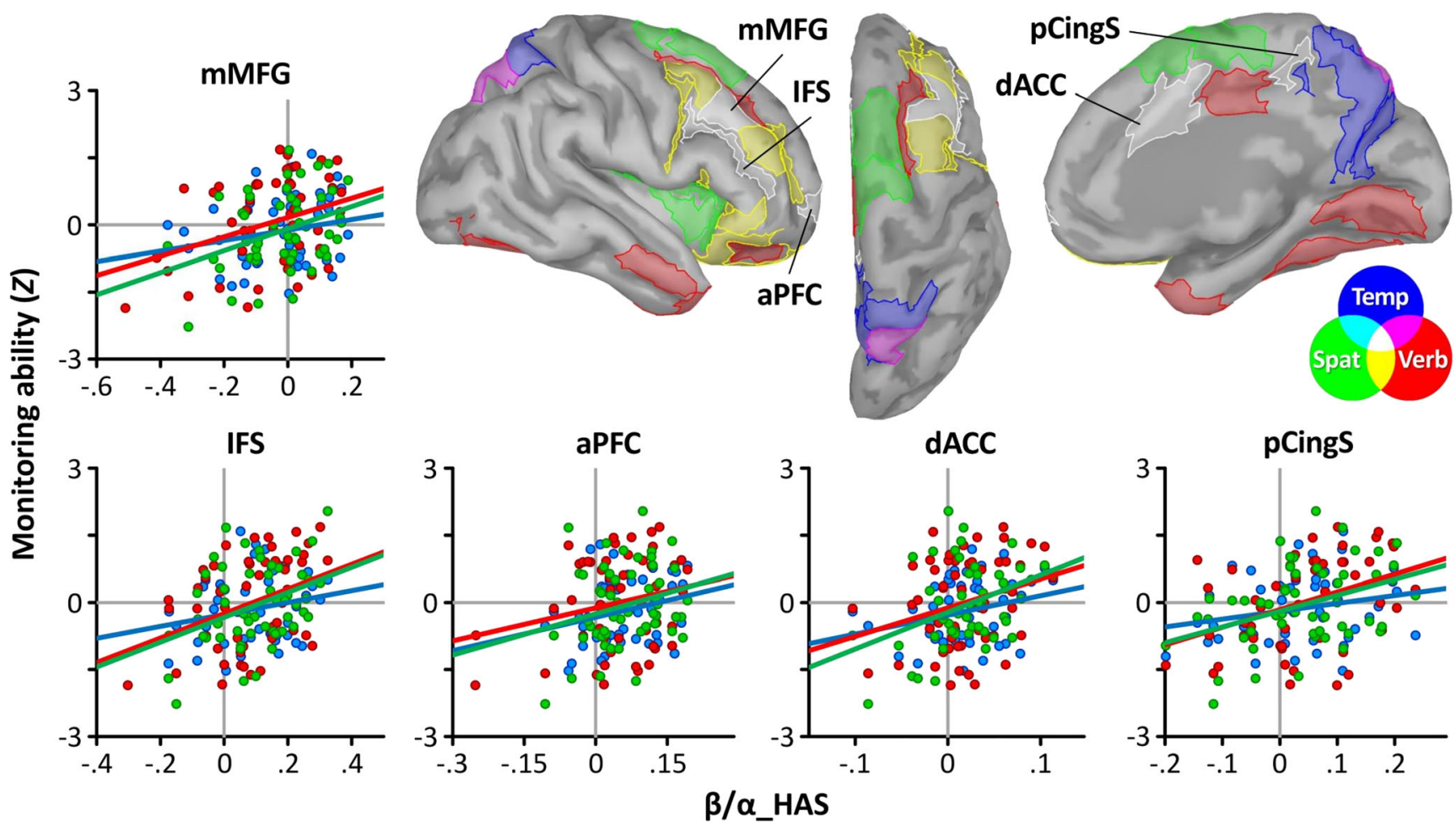

Fig. 3. Results of the power - behavior robust correlation analyses. The cortical surface of the right hemisphere is shown with the regions identified by the intersection analysis as those showing a significant powerbehavior correlation in all the three monitoring tasks; these regions are indicated in white and labeled. The regions showing a significant correlation specifically for the temporal, verbal, and spatial domains are indicated in blue, red, and green, respectively, whereas those showing a significant correlation for two tasks are indicated in yellow, cyan, and magenta (see the color legend on the right). The scatterplots show the corresponding correlations between the participants' hemispheric

asymmetry of rsEEG spectral activity ( $\beta / \alpha$ HAS, $x$ axis) in those regions and the $z$-transformed behavioral measures of their monitoring abilities $(y$ axis) in the temporal, verbal, and spatial domains (blue, red, and green points, respectively). Positive values of $\beta / \alpha \_$HAS indicate a stronger right-lateralized brain activity at rest (see Resting State EEG Recording and Analysis section). The corresponding regression lines reflect the skipped Pearson correlation for each domain. mMFG, middle part of the middle frontal gyrus; IFS, inferior frontal sulcus; aPFC, anterior prefrontal cortex; dACC, dorsal anterior cingulate cortex; pCingS, posterior cingulate sulcus.

interindividual difference in participants' performance can be explained, at least in part, by interindividual differences in the lateralization of intrinsic PFC spectral activity. Specifically, participants with a more left-lateralized resting-state activity in PFC, as indexed by the asymmetry in $\beta / \alpha$ power ratio, had

better criterion-setting abilities, as indexed by reduced switching costs and Stroop effects (Ambrosini \& Vallesi, 2016, 2017). We complemented these findings by testing whether the monitoring function is instead more related to the right lateralization of intrinsic brain dynamics. This

Table 2. Results of the intersection analysis. Cortical regions that showed significant correlations between EEG asymmetries and performance for all the three monitoring tasks

\begin{tabular}{|c|c|c|c|c|c|c|c|c|c|}
\hline \multirow[t]{2}{*}{ Region } & \multicolumn{3}{|c|}{ Temporal monitoring } & \multicolumn{3}{|c|}{ Verbal monitoring } & \multicolumn{3}{|c|}{ Spatial monitoring } \\
\hline & $r_{s k i}$ & ${ }_{L B} C I_{95 \%}$ & ${ }_{U B} C I_{95 \%}$ & $r_{s k i}$ & ${ }_{L B} C I_{95 \%}$ & ${ }_{U B} C I_{95 \%}$ & $r_{s k i}$ & ${ }_{L B} C I_{95 \%}$ & ${ }_{U B} C I_{95 \%}$ \\
\hline mMFG & 0.236 & 0.021 & 0.461 & 0.338 & 0.049 & 0.556 & 0.362 & 0.104 & 0.577 \\
\hline IFS & 0.224 & 0.004 & 0.438 & 0.369 & 0.125 & 0.568 & 0.384 & 0.109 & 0.597 \\
\hline $\mathrm{aPFC}^{\mathrm{a}}$ & 0.258 & 0.014 & 0.505 & 0.229 & 0.006 & 0.453 & 0.247 & 0.001 & 0.511 \\
\hline $\mathrm{dACC}$ & 0.27 & 0.035 & 0.485 & 0.273 & 0.033 & 0.482 & 0.393 & 0.13 & 0.609 \\
\hline pCingS & 0.232 & 0.028 & 0.452 & 0.389 & 0.154 & 0.601 & 0.393 & 0.181 & 0.568 \\
\hline
\end{tabular}

${ }^{a}$ This result did not survive Family-wise error correction for multiple comparisons.

Note: $r_{\text {ski }}$, skipped Pearson's correlation; ${ }_{L B} C I_{95 \%}$ and ${ }_{U B} C I_{95 \%}$, lower and upper bound of the $95 \%$ bootstrap confidence interval, respectively; mMFG, middle part of the middle frontal gyrus; IFS, inferior frontal sulcus; aPFC, anterior prefrontal cortex; dACC, dorsal anterior cingulate cortex; pCingS, posterior cingulate sulcus. All the reported correlations were significant at the nonparametric percentile bootstrap test (see Section 2.5). 
reverse pattern of lateralization would be indeed in line with what suggested by the ROtman-Baycrest Battery to Investigate Attention (ROBBIA) model (see Shallice \& Cipolotti, 2018; Stuss, 2011; Stuss \& Alexander, 2007; Vallesi, 2012, for reviews), which proposes a left and right hemispheric specialization of prefrontal cortex and related neural circuits for, respectively, criterion-setting and monitoring cognitive control processes. To test this prediction, we capitalized on the same approach as in Ambrosini and Vallesi $(2016,2017)$ by calculating the right-left hemispheric asymmetry score for the $\beta / \alpha$ power ratio as a measure of the participants' lateralized brain dynamics at rest. Given that we were interested in the domain-general nature of monitoring, three different cognitive domains (temporal, verbal, and spatial) were used here, but only the results from the intersection analyses highlighting the commonalities between the three tasks will be discussed. Such analyses showed that enhanced monitoring abilities were, indeed, significantly associated with more right-lateralized $\beta / \alpha$ power ratio in different $\mathrm{PFC}$ regions, including the $\mathrm{mMFG}$ and the adjacent IFS, as well as in a dorso-medial region of the parietal lobe.

Regarding the involvement of the $\mathrm{mMFG}$, the same area was previously found to show a significant power-behavior correlation for the criterion-setting performance but with an opposite direction of lateralization, as mentioned earlier (i.e., smaller switching costs and Stroop effects associated with higher $\beta / \alpha$ power in the left mMFG; Ambrosini \& Vallesi, $2016,2017)$. Our differential correlation analysis indeed confirmed that there is a significant bivalent association between the $\beta / \alpha$ HAS at rest in the mMFG and both the monitoring and criterion-setting control processes. More interestingly, the right lateralization of intrinsic brain activity in the very same PFC area was associated with better participants' ability to exert sustained cognitive control (i.e., smaller mixing costs; Ambrosini \& Vallesi, 2016), an executive process that is thought to be also related to monitoring functioning (Vallesi, 2012). In a task-switching paradigm, indeed, mixing costs refer to the performance difference between the repeat trials from the mixed block and the (all) repeat trials from the singletask block. They are generally thought to reflect the operation of continuous processes in charge of maintaining and managing multiple task-sets active throughout the mixed block (Braver et al., 2003; Rubin \& Meiran, 2005; Tarantino, Mazzonetto, \& Vallesi, 2016). As such, mixing costs would be a proxy of the (dys-)functioning of sustained monitoring processes. Again, our differential correlation analysis suggested that the power-behavior correlations between the $\beta / \alpha$ HAS at rest in the mMFG and different behavioral measures of monitoring ability are not statistically different. Together, these findings indicate that the lateralization of the intrinsic PFC spectral activity is differentially related to both criterion-setting and monitoring functions, in line with the hypothesis of a left-right prefrontal hemispheric specialization for these functions (Shallice \& Cipolotti, 2018; Stuss \& Alexander, 2007; Fleck et al., 2006; Reverberi et al., 2005). It is important to note that our behavioral results suggested the independence between behavioral measures of monitoring and criterion-setting abilities, in line with recent confirmatory factor analysis evidence indicating that they are distinct, unrelated executive functions (Ambrosini et al., 2019).

The present results support our hypothesis that interindividual differences in intrinsic prefrontal spectral activity would reflect a stable individual trait denoting a relative propensity or aptitude in engaging a distinct brain network and the related executive function, a factor that would account for the heterogeneity in executive performance. Specifically, individuals with stronger left-lateralized PFC $\beta / \alpha$ at rest might preferentially engage the left (prefrontal nodes of the) frontoparietal brain network mediating criterion-setting processes, whereas those with stronger right-lateralized PFC $\beta / \alpha$ at rest might preferentially engage the right (prefrontal nodes of the) fronto-parietal brain network mediating monitoring processes. The functional role of the hemispheric lateralization in the intrinsic PFC spectral profile would thus be to provide endogenous constraints, or priors, to task-related activity in specific neural networks mediating cognitive processes required by specific tasks (Spadone et al., 2015; Raichle, 2011). Supporting this idea, it has been shown that interindividual variability in resting state BOLD fluctuations may explain variability in both evoked BOLD fluctuations in task-related cortical regions and behavioral performance in executive function tasks (Mennes et al., 2011; Xu et al., 2014; Zou et al., 2013). Of great importance for our proposal of the functional meaning of our results, it also has been shown that left and right frontoparietal networks are indeed two distinct resting-state networks (Damoiseaux et al., 2006; Meyer et al., 2013) that reflect specific lateralized, task-related networks (Smith et al., 2009). Moreover, another study (Mantini et al., 2013) showed that they are evolutionary novel (i.e., human-specific), suggesting their role in mediating the higher-order cognitive control abilities acquired during evolution that characterize human behavior (Duncan et al., 2000; Dehaene et al., 2003).

The PFC areas showing a relationship between rightlateralization and general monitoring performance, which included the dorso- and ventro-lateral PFC, also have been reported to mediate monitoring processes by a number of fMRI studies involving different cognitive domains with heterogeneous task requirements. For instance, increased activation in right $\mathrm{PFC}$ regions matching our lateral $\mathrm{PFC}$ regions has been found when participants had to monitor regular predictable spatial trajectories compared to random irregular ones (Vallesi \& Crescentini, 2011), presence of nonsalient target with respect to a salient target that popped out in a bottomup fashion during a visual search task (Vallesi, 2014), goal 
progress across numerical and visuospatial modalities (Benn et al., 2014), temporal conditional probabilities (Coull et al., 2000; Vallesi et al., 2009), and accumulated evidence in low confidence decision making with both verbal episodic memory retrieval (Henson et al., 2000; Vallesi \& Shallice, 2006) and perceptual tasks (Fleck et al., 2006). Moreover, in a recent simultaneous EEG-fMRI study, the right MFG, along with the right supramarginal gyrus, was more activated when participants had to monitor for target faces as compared to nonmonitoring trials. Such activity also was modulated by the ERPs elicited by monitoring trials over frontal electrodes (Tarantino et al., 2017).

Moreover, the critical causal role of the same PFC areas in monitoring processes has been observed in both neuropsychological and transcranial magnetic stimulation (TMS) studies. Focal damage (Arbula et al., 2017; Vallesi et al., 2007; Stuss et al., 2005) or transient inhibition (Vallesi, Shallice \& Walsh, 2007) of right lateral PFC impairs monitoring of temporal conditional probabilities, thus preventing the usual increase of preparation during longer foreperiods. In line with these findings, patients with lesions in these areas also show difficulties in adjusting their responses to continuous and fast stimulus occurrence (Picton et al., 2006; Shallice et al., 2008), supporting the critical involvement of right lateral PFC in monitoring timed performance. Similar evidence for eventrelated monitoring comes from studies on prospective memory in which both lesions (Uretzky \& Gilboa, 2010) and disruptive stimulations (Bisiacchi et al., 2011) over right PFC interferes with prospective memory cue detection performance which, according to some models (McDaniel \& Einstein, 2000), entails the monitoring process.

These findings are in line with our observation that similar areas in the right PFC support both continuous and eventrelated types of monitoring, regardless of the cognitive domain involved in the task and its specific features. As a further support for this conclusion, two recent studies with fMRI (Nee and D'Esposito, 2016) and theta-burst transcranial magnetic stimulation (Nee \& D'Esposito, 2017) highlighted the role of the MFG as a critical nexus for cognitive control that is involved in integrating bottom-up feature information in lower level posterior motor regions and top-down task information in more rostral prefrontal nodes to guide context-appropriate actions.

It is important to note that, unlike our previous results concerning monitoring-related ability during task-switching (i.e., mixing costs), the present results suggest that the interindividual variability in domain-independent monitoring functioning is related to the hemispheric asymmetries of resting state-related spectral dynamics not only in the lateral PFC, but also in other PFC regions and in the medial parietal cortex. This shows that some degree of task specificity may mediate how domain-general monitoring processes are explained by neurophysiological individual difference factors.
First, the present results indicate that monitoring-related behavioral performance may be mediated by the right lateralization of intrinsic neurophysiological activity in a medial parietal region, the posterior cingulate sulcus. Albeit this region does not belong to the frontoparietal network, it has been shown to participate in both dorsal and ventral attention networks (Yeo et al., 2011; Power et al., 2011) subserving, respectively, goal-directed and stimulus-driven attention processes, for which a right hemispheric asymmetry has been reported. This is consistent with the tight functional relationship between these two distinct attention networks (Corbetta and Shulman, 2002; Corbetta et al., 2008) and suggests that this medial parietal region may be a potential point of interaction between them (Yeo et al., 2014). It is interesting to note that the only other cortical region representing a potential intercommunication node between stimulus-driven and goal-directed attention processes is the right MFG near the IFS (Corbetta et al., 2008; see also Asplund et al., 2010), a lateral PFC region that is also consistent with the mMFG and IFS regions we found here to show a significant power-behavior correlation. This result thus highlights the important role played by attention processes to monitor successfully for the occurrence of taskrelevant critical events (see also Capizzi et al., 2016). Indeed, the ability to focus and maintain attention over time, but also the ability to reorient it when behaviorally relevant stimuli occur, are closely associated with optimal monitoring functioning.

Finally, the present results suggest that monitoring functioning may be mediated by the right lateralization of intrinsic neurophysiological activity in two other PFC regions, a rostral (aPFC) and a medial (dACC) one, albeit the results for the former region did not survive correction for multiple comparisons and should thus be taken with caution. These regions have been shown to be critically involved in monitoringrelated cognitive processes, such as strategic monitoring of prospective cues in prospective memory tasks (Reynolds et al., 2009) for the former area and the detection of discrepancies between the actual state of affairs and the desired state (Benn et al., 2014) for the latter one. More importantly, the above mentioned neuropsychological studies of patients with focal PFC lesions have shown that lesions in the right dACC impair monitoring of temporal probabilities (Stuss et al., 2005) and monitoring of possible discrepancies between patients' behavior and an external temporal reference (Shallice et al., 2008) to optimize their responses according to the task requirements. These findings suggest that the right hemispheric asymmetry in the involvement of PFC in monitoring processes would implicate not only lateral PFC regions, but a more extended cluster of PFC areas also including dACC and aPFC. It is interesting to note that aPFC and $\mathrm{dACC}$ are two nodes of the so-called cingulo-opercolar network, which mediates cognitive processes that are strictly related to 
monitoring functioning, such as the sustained maintenance of the task set (Dosenbach et al. 2006, 2007), as well as of optimal levels of tonic alertness (Sadaghiani \& D'Esposito, 2015) and is involved in detecting important, behaviorally relevant information (Seeley et al., 2007). Taken together with the present study, these findings thus suggest that monitoring functioning relies on the interplay between different cognitive processes mediated by specialized networks.

An interesting issue deserving further investigation is whether the lateralization patterns observed here for monitoring tasks in so-called "cold" cognitive domains is related to models of lateralized prefrontal processes in emotion/ motivation domains. Empirical evidence, for instance, supports an association between right PFC with negative emotions and avoidance motivation as opposed to left PFC involvement in positive emotions and approach motivation, with corresponding brain asymmetries observed both in healthy and psychiatric conditions (Spielberg et al., 2011; Davidson \& Irwin, 1999; Tomarken \& Keener, 1998; Miller, Crocker et al., 2013). Notwithstanding that better efforts should be made to link these two lines of research within the same experimental designs, one could speculate that there are similarities in right lateralized cognitive and emotional processes. It is possible that the right DLPFC and neighboring regions are responsible for monitoring critical events, and as such, negative threats (with related avoidance motivation) could be considered by far among the most critical events that one needs to continuously monitor. Future cross-field research should more directly test this as well as other alternative hypotheses.

\section{Conclusions}

The present EEG study shows that the intrinsic lateralization of brain activity explains the performance in a set of tasks tapping on different aspects of monitoring. In particular, a right lateralized resting-state activity in different nodes belonging to cognitive control and attention networks seems to explain better monitoring performance. Whether these beneficial asymmetries could be modulated through neurostimulation or neuro-feedback to boost performance in the healthy population across the life span or to recover compromised functions in patients is an exciting working hypothesis for future research.

Open practices statement No part of this study was pre-registered prior to the research being conducted. Data and materials will be made available upon request.

Funding information This study was funded by the FP7/2007-2013 European Research Council Starting Grant LEX-MEA to AV [grant number 313692].

\section{Compliance with ethical standards}

Conflict of interest The authors declare that they have no conflict of interest.

Ethical approval The study was approved by the Bioethical Committee of the Azienda Ospedaliera di Padova. All procedures involving human participants were in accordance with the ethical standards of the institutional and/or national research committee and with the 1964 Helsinki declaration and its later amendments or comparable ethical standards.

Informed consent Informed consent was obtained from all individual participants included in the study.

\section{References}

Ambrosini, E., Arbula, S., Rossato, C., Pacella, V., Vallesi, A. (2019). Neuro-cognitive architecture of executive functions: A latent variable analysis. Cortex, 119, 441-456.

Ambrosini, E., \& Vallesi, A. (2016). Asymmetry in prefrontal restingstate EEG spectral power underlies individual differences in phasic and sustained cognitive control. NeuroImage, 124(1), 843-857.

Ambrosini, E., \& Vallesi, A. (2017). Domain-general Stroop performance and hemispheric asymmetries: A Resting-state EEG Study. Journal of Cognitive Neuroscience, 29(5), 769-779.

Arbula, S., Pacella, V., De Pellegrin, S., Rossetto, M., Denaro, L., D'Avella, D., Della Puppa, A., Vallesi, A. (2017). Addressing the selective role of distinct prefrontal areas in response suppression: A study with brain tumor patients. Neuropsychologia, 100, 120-130.

Asplund, C. L., Todd, J. J., Snyder, A. P., \& Marois, R. (2010). A central role for the lateral prefrontal cortex in goal-directed and stimulusdriven attention. Nature Neuroscience, 13(4), 507-512.

Baillet, S., Mosher, J. C., \& Leahy, R. M. (2001). Electromagnetic brain mapping. IEEE Signal Processing Magazine, 18, 14-30.

Benjamini, Y., \& Hochberg, Y. (1995). Controlling the false discovery rate: a practical and powerful approach to multiple testing. Journal of the Royal statistical society: series B (Methodological), 57(1), 289-300.

Benn, Y., Webb, T. L., Chang, B. P., Sun, Y. H., Wilkinson, I. D., \& Farrow, T. F. (2014). The neural basis of monitoring goal progress. Frontiers in Human Neuroscience, 10, 8:688.

Bisiacchi, P., Cona, G., Schiff, S., \& Basso, D. (2011). Modulation of a fronto-parietal network in event-based prospective memory: an rTMS study. Neuropsychologia, 49, 2225-2232.

Braver, T. S., Reynolds, J. R., \& Donaldson, D. I. (2003). Neural mechanisms of transient and sustained cognitive control during task switching. Neuron, 39, 713-726.

Capizzi, M., Ambrosini, E., Arbula, S., Mazzonetto, I., \& Vallesi, A. (2016). Testing the domain-general nature of monitoring in the spatial and verbal cognitive domains. Neuropsychologia, 89, 83-95.

Capizzi, M., \& Correa, A. (2018). Measuring temporal preparation. In Vatakis, A., Balci, F., Di Luca, M. \& Correa, A. (Eds.), Timing and time perception: Procedures, measures, and applications. Brill: Leiden, The Netherlands

Cohen, J. (1977). Statistical power analysis for the behavioral sciences (rev. ed.). Hillsdale, NJ: Lawrence Erlbaum Associates, Inc.

Corbetta, M., Patel, G., \& Shulman, G. L. (2008). The reorienting system of the human brain: from environment to theory of mind. Neuron, 58(3), 306-324.

Corbetta, M., \& Shulman, G. L. (2002). Control of goal-directed and stimulus-driven attention in the brain. Nature Review Neuroscience, 3(3), 201-215. 
Coste, C. P., Sadaghiani, S., Friston, K. J., \& Kleinschmidt, A. (2011). Ongoing brain activity fluctuations directly account for intertrial and indirectly for intersubject variability in Stroop task performance. Cerebral Cortex, 21(11), 2612-2619.

Coull, J. T. (2009). Neural substrates of mounting temporal expectation. PLoSBiology, 7(8), e1000166.

Coull, J. T., Frith, C. D., Büchel, C., Nobre, A. C. (2000). Orienting attention in time: behavioural and neuroanatomical distinction between exogenous and endogenous shifts. Neuropsychologia, 38, 808-819.

Damoiseaux, J. S., Rombouts, S., Barkhof, F., Scheltens, P., Stam, C. J., Smith, S. M., \& Beckmann, C. F. (2006). Consistent resting-state networks across healthy subjects. Proc. Natl. Acad. Sci. U.S.A., 103, 1348-13853.

Davidson, R.J. \& Irwin, W. (1999). The functional neuroanatomy of emotion and affective style. Trends in Cognitive Sciences, 3, 11-21.

Dehaene, S., Piazza, M., Pinel, P., \& Cohen, L. (2003). Three parietal circuits for number processing. Cognitive Neuropsychology, 20(3), 487-506.

Delorme, A., \& Makeig, S. (2004). EEGLAB: an open source toolbox for analysis of single-trial EEG dynamics including independent component analysis. Journal of Neuroscience Methods, 134, 9-21.

Delorme, A., Sejnowski, T., \& Makeig, S. (2007). Enhanced detection of artifacts in EEG data using higher-order statistics and independent component analysis. NeuroImage, 34, 1443-1449.

Destrieux, C., Fischl, B., Dale, A., \& Halgren, E. (2010). Automatic parcellation of human cortical gyri and sulci using standard anatomical nomenclature. NeuroImage, 53, 1-15.

Dosenbach, N. U., Fair, D. A., Miezin, F. M., Cohen, A. L., Wenger, K. K., ... Petersen, S. E. (2007). Distinct brain networks for adaptive and stable task control in humans. Proc Natl Acad Sci USA, 104(26), 11073-11078.

Dosenbach, N. U., Visscher, K. M., Palmer, E. D., Miezin, F. M., Wenger, K. K. ... Petersen, S. E. (2006). A core system for the implementation of task sets. Neuron, 50(5), 799-812.

Duncan, J., Seitz, R. J., Kolodny, J., Bor, D., Herzog, H., Ahmed, A., ... Emslie, H. (2000). A neural basis for general intelligence. Science, 289(5478), 457-460.

Fiser, J., Berkes, P., Orbàn, G., \& Lengyel, M. (2010). Statistically optimal perception and learning: from behavior to neural representations. Trends in Cognitive Sciences, 14(3), 119-130.

Fleck, M. S., Daselaar, S. M., Dobbins, I. G., \& Cabeza, R. (2006). Role of prefrontal and anterior cingulate regions in decision-making processes shared by memory and nonmemory tasks. Cerebral Cortex 16(11), 1623-1630.

Fryer, S. L., Roach, B. J., Ford, J. M., Turner, J. A., van Erp, T. G., ... Mathalon, D. H. (2015). Relating intrinsic low-frequency BOLD cortical oscillations to cognition in schizophrenia. Neuropsychopharmacology, 40(12), 2705-2714.

Gordon, S., Todder, D., Deutsch, I., Garbi, D., Getter, N., \& Meiran, N. (2018). Are resting state spectral power measures related to executive functions in healthy young adults? Neuropsychologia, 108, $61-72$.

Gramfort, A., Papadopoulo, T., Olivi, E., \& Clerc, M. (2010). OpenMEEG: Opensource software for quasistatic bioelectromagnetics. Biomedical Engineering Online, 9, 45.

Harmelech, T., \& Malach, R. (2013). Neurocognitive biases and the patterns of spontaneous correlations in the human cortex. Trends in cognitive sciences, 17(12), 606-615.

Henson, R. N., Rugg, M. D., Shallice, T., Dolan, R. J. (2000). Confidence in recognition memory for words: dissociating right prefrontal roles in episodic retrieval. J Cogn Neurosci, 12, 913-923.

Jahanshahi, M., Profice, P., Brown, R. G., Ridding, M. C., Dirnberger, G., \& Rothwell, J. C. (1998). The effects of transcranial magnetic stimulation over the dorsolateral prefrontal cortex on suppression of habitual counting during random number generation. Brain, 121(8), 1533-1544.

Kilner, J. M., Mattout, J., Henson, R., \& Friston, K. J. (2005). Hemodynamic correlates of EEG: a heuristic. NeuroImage, 28, 280-286.

Kybic, J., Clerc, M., Abboud, T., Faugeras, O., Keriven, R., Papadopoulo, T. (2005). A common formalism for the integral formulations of the forward EEG problem. IEEE Transaction on Medical Imaging 24, 12-28.

Laufs, H., Holt, J. L., Elfont, R., Krams, M., Paul, J. S., Krakow, K., \& Kleinschmidt, A. (2006). Where the BOLD signal goes when alpha EEG leaves. NeuroImage, 31, 1408-1418.

Laufs, H. (2008). Endogenous brain oscillations and related networks detected by surface EEG-combined fMRI. Human Brain Mapping, $29,762-769$

Mantini, D., Corbetta, M., Romani, G. L., Orban, G. A., \& Vanduffel, W. (2013). Evolutionarily novel functional networks in the human brain? Journal of Neuroscience, 33(8), 3259-3275.

Mantini, D., Perrucci, M. G., Del Gratta, C., Romani, G. L. \& Corbetta, M. (2007). Electrophysiological signatures of resting state networks in the human brain. Proc Natl Acad. Sci. USA, 104, 13170-13175.

McDaniel, M. A., \& Einstein, G. O. (2000). Strategic and automatic processes in prospective memory retrieval: a multiprocess framework. Applied Cognitive Psychology, 14, 127-144.

Mennes, M., Zuo, X. N., Kelly, C., Di Martino, A., Zang, Y. F., Biswal, B., Castellanos, F. X., Milham, M. P. (2011). Linking interindividual differences in neural activation and behavior to intrinsic brain dynamics. NeuroImage, 54, 2950-2959.

Meyer, M. C., van Oort, E. S., \& Barth, M. (2013). Electrophysiological correlation patterns of resting state networks in single subjects: a combined EEG-fMRI study. Brain Topography, 26, 98-109.

Miller, G. A., Crocker, L. D., Spielberg, J. M., Infantolino, Z. P. \& Heller, W. (2013). Issues in localization of brain function: The case of lateralized frontal cortex in cognition, emotion, and psychopathology. Front Integr Neurosci, 7, 2.

Nee, D. E., \& D'Esposito, M. (2016). The hierarchical organization of the lateral prefrontal cortex. eLife, 5 .

Nee, D. E., \& D'Esposito, M. (2017). Causal evidence for lateral prefrontal cortex dynamics supporting cognitive control. eLife, 6 .

Niemi, P., \& Näätänen, R. (1981). Foreperiod and simple reaction time. Psychological Bulletin, 89(1), 133-162.

Nobre, A. C., Correa, A., \& Coull, J. T. (2007). The hazards of time. Current opinion in neurobiology, 17(4), 465-470.

Oldfield, R. C. (1971). The assessment and analysis of handedness: the Edinburgh inventory. Neuropsychologia, 9(1), 97-113.

Ota, T., Toyoshima, R., \& Yamauchi, T. (1996). Measurements by biphasic changes of the alpha band amplitude as indicators of arousal level. International Journal of Psychophysiology, 24, 25-37.

Pernet, C. R., Wilcox, R., \& Rousselet, G. A. (2012). Robust correlation analyses: false positive and power validation using a new open source matlab toolbox. Frontiers in Psychology, 3, 606.

Perrin, F., Pernier, J., Bertrand, O., \& Echallier, J. F. (1989). Spherical splines for scalp potential and current density mapping. Electroencephalography and Clinical Neurophysiology, 72(2), 184-187.

Picton, T. W., Stuss, D. T., Shallice, T., Alexander, M. P., \& Gillingham, S. (2006). Keeping time: Effects of focal frontal lesions. Neuropsychologia, 44, 1195-1209.

Power, J. D., Cohen, A. L., Nelson, S. M., Wig, G. S., Barnes, K. A., .. Petersen, S. E. (2011). Functional network organization of the human brain. Neuron, 72(4), 665-678.

Raichle, M. E. (2011). The restless brain. Brain Connectivity, 1, 3-12.

Reverberi, C., Lavaroni, A., Gigli, G. L., Skrap, M., \& Shallice, T. (2005). Specific impairments of rule induction in different frontal lobe subgroups. Neuropsychologia, 43, 460-72. 
Reynolds, J. R., West, R., \& Braver, T. (2009). Distinct neural circuits support transient and sustained processes in prospective memory and working memory. Cerebral Cortex, 19(5), 1208-1221.

Rosa, M. J., Kilner, J., Blankenburg, F., Josephs, O., \& Penny, W. (2010). Estimating the transfer function from neuronal activity to BOLD using simultaneous EEG-fMRI. NeuroImage, 49, 1496-1509.

Rousseeuw, P. J., \& Verboven, S. (2002). Robust estimation in very small samples. Computational Statistics \& Data Analysis, 40, 741-758.

Rubin, O., Meiran, N. (2005). On the origins of the task mixing cost in the cuing task-switching paradigm. Journal of Experimental Psychology: Learning, Memory, and Cognition, 31, 1477-1491.

Sadaghiani, S., \& D'Esposito, M. (2015). Functional characterization of the cingulo-opercular network in the maintenance of tonic alertness. Cerebral Cortex, 25(9), 2763-2773.

Sadaghiani, S., \& Kleinschmidt, A. (2013). Functional interactions between intrinsic brain activity and behavior. NeuroImage, 80 , 379-386.

Schneider, W., Eschman, A., \& Zuccolotto, A. (2002). E-Prime user's guide. Pittsburgh: Psychology Software Tools Inc.

Seeley, W. W., Menon, V., Schatzberg, A. F., Keller, J., Glover, G. H., ... Greicius, M. D. (2007). Dissociable intrinsic connectivity networks for salience processing and executive control. Journal of Neuroscience, 27(9), 2349-2356.

Shallice, T., \& Cipolotti, L. (2018). Prefrontal cortex and neurological impairments of active thought. Annual Review of Psychology, 69:1.

Shallice, T., Stuss, D. T., Picton, T. W., Alexander, M. P., \& Gillingham, S. (2007). Multiple effects of prefrontal lesions on task-switching. Frontiers in Human Neuroscience, 1, 2.

Shallice, T., Stuss, D. T., Picton, T. W., Alexander, M. P., \& Gillingham, S. (2008). Mapping task switching in frontal cortex through neuropsychological group studies. Frontiers in Neuroscience, 2, 79-85.

Smith, S. M., Fox, P. T., Miller, K. L., Glahn, D. C., Fox, P. M., Mackay, C. E., Filippini, N., Watkins, K. E., Toro, R., Laird, A. R., \& Beckmann, C. F. (2009). Correspondence of the brain's functional architecture during activation and rest. Proc. Natl. Acad. Sci. U.S.A., 106, 13040-13045.

Spadone, S., Della Penna, S., Sestieri, C., Betti, V., Tosoni, A., Perrucci, M. G., ... \& Corbetta, M. (2015). Dynamic reorganization of human resting-state networks during visuospatial attention. Proceedings of the National Academy of Sciences, 112(26), 8112-8117.

Spielberg, J. M., Miller, G. A., Engels, A. S., Herrington, J.D., Sutton, B. P., Banich, M. T. \& Heller, W. (2011). Trait approach and avoidance motivation: lateralized neural activity associated with executive function. Neuroimage, 54(1), 661-70

Steiger, J. H. (1980). Tests for Comparing Elements of a Correlation Matrix. Psychol Bull, 87, 245-251.

Steinborn, M. B., \& Langner, R. (2012). Arousal modulates temporal preparation under increased time uncertainty: Evidence from higher-order sequential foreperiod effects. Acta Psychologica, 139, 65-76.

Stuss, D. T. (2011). Functions of the frontal lobes: relation to executive functions. Journal of the International Neuropsychological Society, 17, 759-765

Stuss, D. T., \& Alexander, M. P. (2007). Is there a dysexecutive syndrome? Philosophical Transactions of the Royal Society, 362, 901-915.

Stuss, D. T., Alexander, M. P., Shallice, T., Picton, T. W., Binns, M. A., Macdonald, R., Borowiec, A., \& Katz, D. I. (2005). Multiple frontal systems controlling response speed. Neuropsychologia, 43, 396-417.

Tadel, F., Baillet, S., Mosher, J. C., Pantazis, D., \& Leahy, R. M. (2011). Brainstorm: A user-friendly application for MEG/EEG analysis. Computational Intelligence and Neuroscience, 2011, 879716.

Tarantino, V., Mazzonetto, I., Formica, S., Causin, F., \& Vallesi, A. (2017). The neural bases of event monitoring across domains: a simultaneous ERP-fMRI study. Frontiers in Human Neuroscience, 11:376.

Tarantino, V., Mazzonetto, I., \& Vallesi, A. (2016). Electrophysiological correlates of the cognitive control processes underpinning mixing and switching costs. Brain Research, 1646, 160-173.

Tomarken, A. J. \& Keener, A.D. (1998). Frontal brain asymmetry and depression: A self-regulatory perspective. Cognition and Emotion, $12,387-420$

Uretzky, S., \& Gilboa, A. (2010). Knowing your lines but missing your cue: rostral prefrontal lesions impair prospective memory cue detection, but not action-intention superiority. Journal of Cognitive Neuroscience, 22(12), 2745-2757.

Vallesi A. (2010). Neuroanatomical substrates of foreperiod effects. In A.C. Nobre, J.T. Coull (Eds.), Attention and time (pp. 303-316). Oxford: Oxford University Press.

Vallesi, A. (2012). Organization of executive functions: hemispheric asymmetries. Journal of Cognitive Psychology, 24(4), 367-386.

Vallesi, A. (2014). Monitoring mechanisms in visual search: an fMRI study. Brain Research, 1579, 65-73.

Vallesi, A., Arbula, S., \& Bernardis, P. (2014). Functional dissociations in temporal preparation: evidence from dual-task performance. Cognition, 130(2), 141-151.

Vallesi, A., \& Crescentini, C. (2011). Right fronto-parietal involvement in monitoring spatial trajectories. Neurolmage, 57, 558-564.

Vallesi, A., McIntosh, A. R., Shallice, T., \& Stuss, D. T. (2009). When time shapes behavior: fMRI evidence of brain correlates of temporal monitoring. Journal of Cognitive Neuroscience, 21(6), 1116-1126.

Vallesi, A., \& Shallice T. (2006). Prefrontal involvement in source memory: an electrophysiological investigation of accounts concerning confidence and accuracy. Brain Res, 1124(1), 111-125.

Vallesi, A., \& Shallice, T. (2007). Developmental dissociations of preparation over time: Deconstructing the variable foreperiod phenomena. Journal of Experimental Psychology. Human Perception and Performance, 33(6), 1377-1388.

Vallesi, A., Mussoni, A., Mondani, M., Budai, R., Skrap, M., \& Shallice, T. (2007). The neural basis of temporal preparation: insights from brain tumor patients. Neuropsychologia, 45(12), 2755-2763.

Vallesi, A., Lozano, V. N., Correa, A. (2013). Dissociating temporal preparation processes as a function of the inter-trial interval duration. Cognition, 127(1), 22-30.

van Dam, W. O., Decker, S. L., Durbin, J. S., Vendemia, J. M., \& Desai, R. H. (2015). Resting state signatures of domain and demandspecific working memory performance. NeuroImage, 118, 174-182.

Verboven, S., \& Hubert, M. (2005). LIBRA: a MATLAB library for robust analysis. Chemometrics and Intelligent Laboratory Systems, $75,127-136$.

Verboven, S., \& Hubert, M. (2010). Matlab library LIBRA. Wiley Interdiscip. Review Computational Statistics, 2, 509-515.

Visalli, A., \& Vallesi, A. (2018). Monitoring processes in visual search enhanced by professional experience: The case of orange qualitycontrol workers. Frontiers in psychology, 9:145.

Wang, X., Han, Z., He, Y., Caramazza, A., Song, L., \& Bi, Y. (2013). Where color rests: Spontaneous brain activity of bilateral fusiform and lingual regions predicts object color knowledge performance. NeuroImage, 76, 252-263.

Wilcox, R. (2004). Inferences based on a skipped correlation coefficient. Journal of Applied Statistics, 31(2), 131-143.

Wong, C. W., Olafsson, V., Plank, M., Snider, J., Halgren, E., Poizner, H., \& Liu, T. T. (2014). Resting-state fMRI activity predicts unsupervised learning and memory in an immersive virtual reality environment. PLoS One, 9(10): e109622.

World Medical Association (2013). World Medical Association Declaration of Helsinki: ethical principles for medical research involving human subjects. JAMA 310, 2191-2194. 
Xu, M., De Beuckelaer, A., Wang, X., Liu, L., Song, Y., \& Liu, J. (2015). Regional amplitude of the low-frequency fluctuations at rest predicts word-reading skill. Neuroscience, 298, 318-328.

Xu, J., Rees, G., Yin, X., Song, C., Han, Y., Ge, H., Pang, Z., Xu, W, Tang, Y., Friston, K., \& Liu, S. (2014). Spontaneous neuronal activity predicts intersubject variations in executive control of attention. Neuroscience, 263, 181-192.

Yeo, B. T., Krienen, F. M., Sepulcre, J., Sabuncu, M. R., Lashkari, D., Hollinshead, M., \& Fischl, B. (2011). The organization of the human cerebral cortex estimated by intrinsic functional connectivity. Journal of neurophysiology, 106(3), 1125-1165.

Yeo, B. T., Krienen, F. M., Chee, M. W., \& Buckner, R. L. (2014). Estimates of segregation and overlap of functional connectivity networks in the human cerebral cortex. NeuroImage, 88, 212-227.
Yin, S., Zhu, X., He, R., Li, R. \& Li, J. (2015). Spontaneous activity in the precuneus predicts individual differences in verbal fluency in $\operatorname{cog}$ nitively normal elderly. Neuropsychology, 29(6), 961-970.

Zou, G.Y. (2007). Toward Using Confidence Intervals to Compare Correlations. Psychol Methods, 12, 399-413.

Zou, Q., Ross, T. J., Gu, H., Geng, X., Zuo, X. N., ... Yang, Y. (2013). Intrinsic resting-state activity predicts working memory brain activation and behavioural performance. Human Brain Mapping, 34(12), 3204-3215.

Publisher's note Springer Nature remains neutral with regard to jurisdictional claims in published maps and institutional affiliations. 